\title{
Preeclampsia como factor de riesgo de parálisis cerebral. Estudio de casos y controles
}

\author{
Mirta N. Mesquita-Ramírez ${ }^{1,2 *}$, Laura Evangelina-Godoy ${ }^{1,2}$ y Graciela Leguizamón $n^{1,2}$ \\ ${ }^{1}$ Departamento de Neuropediatría; ${ }^{2}$ Departamento de Pediatría Ambulatoria. Hospital General Pediátrico Niños de Acosta Nu, San Lorenzo, Paraguay
}

\section{Resumen}

Introducción: La parálisis cerebral (PC) es una entidad multifactorial, frecuentemente relacionada con factores de riesgo perinatales. El objetivo del presente estudio fue determinar la asociación entre preeclampsia materna y PC en una población pediátrica hospitalaria. Material y métodos: Estudio de casos y controles. Los casos fueron pacientes con PC del dêpartamento de neuropediatría y los controles, pacientes de la consulta ambulatoria. Los datos fueron recogidos por medio de entrevistas con la madre y consulta de historias clínicas del periodo neonatal. Variables: complicaciones del embarazōy el parto, antropometría neonatal, edad gestacional e historia de hospitalización en la unidad de cuidados intensivos neonatăles. Los datos fueron analizados con SPSSv21, con análisis univariado y regresión logística binaria. El estudio fue aprobadopor el Comité de ética institucional con consentimiento informado. Resultados: Ingresaron 243 pacientes, 81 casos y 162 controles. En el análisis univariado, los antecedentes neonatales de bajo peso de nacimiento, prematuridad, ingreso a la unidad de cuidados intensivos neonatales, luminoterapia, Apgar menor a 5 a los $5 \mathrm{~min}, y$ los antecedentes maternos de preeclampsia, e infecciones durante en el embarazo se asociaron a PC. En el análisis multivariado, la PC se asoció a la preeclampsia (OR: 5.6; IC 95\%: 2-15; $p$ < 0.001) y antecedente de ingreso a la unidad de cuidados intensivos neonatales (OR: 7.3; IC 95\%: 2-24). Conclusiones: La PC se asoció con antecedentes maternos de preeclampsia durante el embarazo y con los antecedentes neonatales de ingreso a la unidad de cuidados intensivos neonatales.

Palabras clave: Parálisis cerebral. Factores de riesgo. Preeclampsia. Unidad de cuidados intensivos neonatales.

\section{Preclampsia as a risk factor for cerebral palsy. Cases and controls study}

\section{Abstract}

Introduction: Cerebral palsy (CP) is a multifactorial entity, frequently related to perinatal risk factors. The goal of the study was to determine the association between maternal preeclampsia and cerebral palsy in a pediatric hospital based population. Material and methods: Cases and controls study. The cases were patients with PC of the neuropediatric department and the controls, outpatiens. Data were collected through interviews with the mother and consultation of clinical records of the neonatal period. Variables: complications of pregnancy and childbirth, neonatal anthropometry, gestational age, and admission to the neonatal intensive care unit. The data were analyzed with SPSSV21, using univariate analysis and binary logistic regression. The study was approved by the institutional review board with informed consent. Results: 243 patients were included, 81 cases and 162 controls. In the univariate analysis, the neonatal antecedents of low birth weight, prematurity, admission to the neonatal intensive care unit, luminotherapy, Apgar score $<5$ at $5 \mathrm{~min}$ and the maternal diagnosis of

\section{Correspondencia:}

*Mirta N. Mesquita-Ramírez

E-mail: mirtanmr@gmail.com

0187-5337/@ 2019. Instituto Nacion licencia CC BY-NC-ND (http://creativecommons.org/licenses/by-nc-nd/4.0/).
Fecha de recepción: 02-07-2019

Fecha de aceptación: 07-11-2019

DOI: 10.24875/PER.19000054
Disponible en internet: 18-12-2019 Perinatol Reprod Hum. 2019;33:66-71 www.perinatologia. $m x$ 
preeclampsia, and infections during pregnancy were associated with CP. In the multivariate analysis, CP was associated with preeclampsia (OR: 5.6; 95\% confidence interval [Cl]: 2-15; $p<0.001$ ) and admission to the neonatal intensive care unit (OR: 7.3: 95\% Cl: 2-24). Conclusions: CP was associated with maternal history of preeclampsia during pregnancy and with neonatal history of admission to the neonatal intensive care unit.

Key words: Cerebral palsy. Risk factors. Preeclampsia. Neonatal intensice neonatal care.

\section{Introducción}

Actualmente se define la parálisis cerebral (PC) como un grupo heterogéneo de alteraciones permanentes del movimiento y la postura que produce limitación de la actividad. No es progresiva y se origina en alteraciones ocurridas en el cerebro del feto 0 en el periodo neonatal inmediato. A menudo se acompaña de alteraciones de la sensación, la percepción, la cognición, la comunicación, la conducta, epilepsia y problemas musculoesqueléticos secundarios ${ }^{1}$. La prevalencia global de la PC es de 2.11 por 1,000 nacidos vivos. Teniendo en cuenta 138 millones de nacidos vivos en todo el mundo, podría considerarse como una importante causa de discapacidad ${ }^{2}$. Sin embargo, tanto la incidencia como los factores de riesgo varían de una región a otra del mundo ${ }^{3}$. Los factores de riesgo de la PC son varios, y en muchos de ellos se desconoce cómo se produce la lesión. Hay causas predisponentes, la mayoría de las cuales se relacionan con alteraciones en el periodo perinatal, durante el embarazo, el parto y el periodo neonatal inmediato (prematuridad, bajo peso de nacimiento (BPN) e infecciones) ${ }^{3,4}$. En un tercio de los casos de PC no se identifican factores de riesgo predisponentes, y en este grupo de pacientes se ha estudiado la probable implicación de factores genéticos. Hay cuatro tipos principales de variación del ADN que contribuyen en la patogénesis de la PC. En algunos estudios se encontraron variaciones en el número de copias y mutaciones de los genes que podrían estar relacionadas con diversos tipos de presentación clínica y gravedad ${ }^{5,6}$.

La mayor parte de la información sobre PC proviene de estudios realizados en países desarrollados que cuentan con un buen registro de la información, a diferencia de países menos desarrollados, donde la atención perinatal es deficiente, la mortalidad materna y neonatal son más elevadas y las fuentes de información son escasas. En Paraguay, el 14\% de la población infantil y adolescente tienen algún tipo de discapacidad, cuya causa está relacionada con factores perinatales.

La preeclampsia/eclampsia es prevalente en el país y es la tercera causa de mortalidad materna ${ }^{7}$. El objetivo del presente estudio fue determinar si existe asociación entre el antecedente materno de preeclampsia/eclampsia y los pacientes con PC de un servicio de neuropediatría

\section{Metodología}

\section{Diseño y lugar del estudio}

Estudio de casos y controles realizado en el Hospital General Pediátrico Niños de Acosta Ñu de San Lorênzo (Paraguay), en los meses de junio a setiembre de $2 \underline{\underline{0}} 18$.

\section{Población, criterios de inclusión y tamä} muestral

\section{DEFINICIÓN DE CASOS}

Pacientes con PC, diagnosticada por los neuropédiatras del hospital, y que se encontraban asistidos regularmente por un equipo multidisciplinario en el departamento de neuropediatría. De acuerdo a la áfectación motora se clasificaron en espásticos, distónicicos o coreoatetósicos y formas mixtas.

\section{DEFINICIÓN DE LOS CONTROLES}

Pacientes apareados por edad y procedencia con los casos, que acudieron al consultorio ambulatorio del mismo hospital para control, o por enfermedades lêves que no requirieron hospitalización.

Tanto para los casos como para los controles, criterios de inclusión fueron: contar con los datos perinatales, ya sea a través de las historias clínicas, agenda pediátrica o entrevista con la madre, y tenê el consentimiento informado. Se excluyeron pacieñtes con malformaciones congénitas, síndromes cromōsómicos, enfermedad neuromuscular primaria, e inçonsistencia en los datos referidos por las madres.

Se estudiaron variables demográficas de los pacientes: edad en el momento de la recolección de los datos, sexo y procedencia; los antecedentes del período neonatal: edad gestacional en semanas, peso en gramos y Apgar a los $5 \mathrm{~min}$, de acuerdo a los datos de la agenda pediátrica. La clasificación de acuerdo al peso 
y edad gestacional se calculó utilizando la curva Intergrowth-2 $1^{\text {st }}$. Por medio de una entrevista estructurada con la madre y de datos de la historia clínica en los casos de hospitalización, se obtuvo información sobre los antecedentes de los niños participantes en relación con el ingreso a la unidad de cuidados intensivos neonatales (UCIN) y el diagnóstico de hiperbilirrubinemia que requirió luminoterapia, así como los antecedentes durante el embarazo, como las infecciones, diabetes, preeclampsia e hipertensión arterial crónica.

Se definió como infecciones maternas a episodios de fiebre durante el embarazo, o signos y síntomas urinarios como disuria o polaquiuria con urocultivos positivos, o diagnóstico de toxoplasmosis, citomegalovirus, VIH o sífilis. El antecedente de preeclampsia se estableció mediante el diagnóstico proporcionado a la mujer por el ginecólogo, con síntomas de hipertensión arterial durante el embarazo (sistólica $>140 \mathrm{mmHg}$ y diastólica $>90 \mathrm{mmHg}$ ) acompañados de edema de los miembros inferiores y proteinuria. Se consideró hipertensión arterial crónica al aumento de la presión arterial no relacionada con el embarazo y que requirió tratamiento médico. El diagnóstico de diabetes se basó en la hiperglucemia que requirió tratamiento médico con o sin relación al embarazo. Se estudiaron, además, las variables maternas de edad, paridad y tipo de parto.

Para el cálculo del tamaño de la muestra, se utilizó el estadígrafo zeta, para detectar una diferencia del $22 \%$ en uno de los factores de riesgo de casos y controles, con una potencia del $20 \%$, para un alfa del $5 \%$ bilateral, se necesitó reclutar 81 casos y 162 controles.

\section{Análisis de los datos}

Los datos fueron procesados en SPSS v21. Las variables continuas se expresaron en medias con desvíos estándar o mediana con mínimo y máximo, de acuerdo a su distribución. Las variables cualitativas se expresaron en porcentajes. Para el análisis de las variables continuas, se utilizó la prueba T Student o la U Mann Whitney, según su distribución. La asociación entre variables cualitativas se realizó por la prueba de Chi cuadrado o el Test de Fisher, según necesidad. Para el análisis multivariado se utilizó la regresión logística binaria por el método intro de las variables con significación estadística, en el análisis univariado. Se analizaron las posibles variables de confusión y modificadoras del efecto. La variable dependiente fue la PC, dicotómica, y las principales variables independientes fueron: el antecedente materno de preeclampsia, las infecciones maternas, y el antecedente de ingreso a la
Tabla 1. Características demográficas de la población estudiada $(n=243)$

\begin{tabular}{|c|c|c|c|}
\hline & $\begin{array}{l}\text { Casos } \\
(n=81)\end{array}$ & $\begin{array}{l}\text { Controles } \\
(n=162)\end{array}$ & $\stackrel{\substack{0 \\
d}}{z}$ \\
\hline Edad (años) & & & हृ \\
\hline Mediana (mín-más) & $\begin{array}{c}5(1-12) \\
n(\%)\end{array}$ & $\begin{array}{c}5(1-11) \\
n(\%)\end{array}$ & $0.48 *$ \\
\hline Sexo masculino Procedencia & $50(61.7)$ & $84(51.9)$ & $0.15^{\dagger}$ \\
\hline Departamento central & $61(75.3)$ & $133(82.1)$ & $\dot{\check{C}}$ \\
\hline Otros departamentos & $20(24.7)$ & $20(17.9)$ & 0.28 \\
\hline $\begin{array}{l}\text { *U Mann Whytney. } \\
{ }^{+} \text {Chi cuadrado. }\end{array}$ & & & 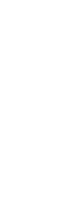 \\
\hline
\end{tabular}

UCIN. El protocolo de estudio fue aprobado por el Comité de ética institucional, con consentimiento informado de los padres o encargados de los pacientes.

\section{Resultados}

Durante el periodo de estudio, 243 pacientes cutmplieron los criterios de inclusión, 81 casos con PO y y 162 controles. Las características de la población en elimomento del reclutamiento se observan en la tabla 1.

En el 42.5\% (107/243) se encontró al menos un fáctor de riesgo perinatal: el $92.6 \%$ (75/81) entre los casos de PC y el 19.8\% (32/162) entre los controles (OR: 50; IC 95\%: 20-127; $p=0.0001)$.

En relación con los antecedentes maternos no hübo diferencias en la edad, paridad y tipo de parto en los casos y controles. Sí se observaron diferencias en la morbilidad, tal como se muestra en la tabla 2.

En el análisis de los antecedentes, en el periodo neonatal se observaron diferencias significativas que se detallan en la tabla 3.

Se realizó el análisis de regresión logística pơr el método intro, incluyendo las variables con diferencias significativas neonatales y de la madre, entre los grupos y los controles. La variable pequeños para la edad gestacional (PEG) y muy bajo peso de nacimiênto (MBPN) no se incluyeron, por el escaso númerö en cada grupo. Las variables peso de nacimiento, edad gestacional y Apgar a los 5 min se dicotomizaron, $y$ se introdujeron así en la ecuación. Los resultados dê la regresión logística se observan en la tabla 4.

\section{Discusión}

En el presente estudio, 6 de cada 10 pacientes con PC fueron recién nacidos a término, y tenían anteceđentes de riesgo perinatal. Estos datos son consistentes 
Tabla 2. Antecedentes del embarazo y del parto de las madres de los participantes $(n=243)$

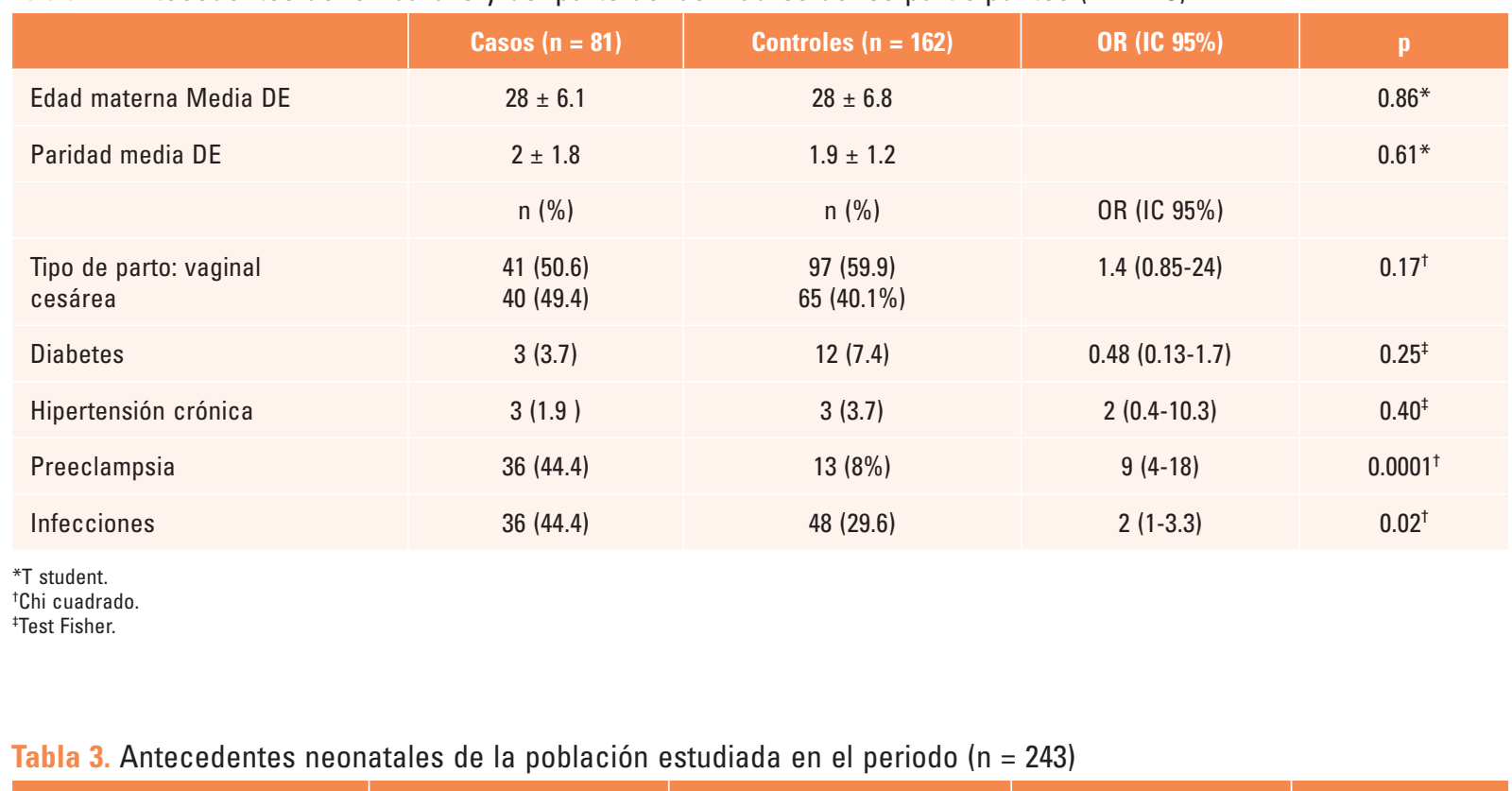

\begin{tabular}{|c|c|c|c|c|c|}
\hline \multirow{2}{*}{$\begin{array}{l}\text { Peso de nacimiento } \\
\text { Media DE }\end{array}$} & Casos (n= 81) & Controles ( $n=162$ ) & & \multicolumn{2}{|l|}{ p } \\
\hline & $2,693 \pm 949$ & $3,257 \pm 554$ & & 0.0001 & $* \frac{0}{0}$ \\
\hline $\begin{array}{l}\text { Edad gestacional } \\
\text { Media DE }\end{array}$ & $36 \pm 4.3$ & $38 \pm 1.4$ & & 0.0001 & * \\
\hline \multirow[t]{2}{*}{$\begin{array}{l}\text { Apgar } 5 \text { min } \\
\text { Media DE }\end{array}$} & $6.9 \pm 2$ & $8.8 \pm 0.47$ & & 0.0001 & * \\
\hline & n (\%) & n (\%) & OR (IC 95\%) & & \\
\hline PEG & $6(7.4)$ & $1(0.6)$ & $12.8(1.5-108)$ & $0.03^{\dagger}$ & $\frac{7}{8}$ \\
\hline BPN & 32 (39.5) & $7(4.3)$ & $14.4(6-34)$ & 0.0001 & $1^{*}$ 읃 \\
\hline MBPN & $10(12.3)$ & $1(0.6)$ & $14(1.6-124)$ & 0.016 & के \\
\hline Apgar $<5$ & $21(25.9)$ & $2(1.2)$ & $28(6.3-123)$ & 0.0001 & $1^{+}$ \\
\hline Prematuridad & $32(39.5)$ & $26(16)$ & $3.4(1.8-6.2)$ & 0.0001 & \\
\hline Ingreso a UCIN & $55(67.9)$ & $10(6.2)$ & $32(14.5-70)$ & 0.0001 & $1^{*}=\frac{\bar{z}}{\mathrm{U}}$ \\
\hline Luminoterapia & $24(29.6)$ & $18(11.1)$ & $3.3(1.7-6.6)$ & 0.0001 & \\
\hline \multicolumn{5}{|c|}{$\begin{array}{l}\text { *T Student. } \\
\text { 'Test Fischer. } \\
\text { 'Chi cuadrado. } \\
\text { BPN: bajo peso de nacimiento; MBPN: muy bajo peso de nacimiento; PEG: pequeño para edad gestacional; UCIN: unidad de cuidados intensivos neonatales. }\end{array}$} & 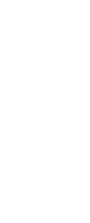 \\
\hline \multicolumn{2}{|c|}{$\begin{array}{l}\text { con lo reportado en la literatura y con la prevalencia de } \\
\text { factores perinatales de riesgo como antecedentes } \\
\text { de } \mathrm{PC}^{4} \text {. En la India, en un estudio sobre prevalencia de } \\
\text { trastornos del neurodesarrollo, el } 72 \% \text { de los casos de } \\
\text { PC se asoció a factores perinatales }{ }^{8} \text {. Las enfermedades } \\
\text { maternas durante el embarazo fueron } 3 \text { veces más fre- } \\
\text { cuentes en pacientes con PC en un estudio realizado } \\
\text { en una región de China }{ }^{9} \text {. La mejora de los cuidados } \\
\text { neonatales y la mayor sobrevida de los prematuros y el }\end{array}$} & \multicolumn{4}{|c|}{$\begin{array}{l}\text { MBPN se acompañó de una disminución de la inciden } \\
\text { cia de PC, lo que fortalece la relación causal entré } \\
\text { factores perinatales y la } \mathrm{PC}^{10} \text {. } \\
\text { En relación con la asociación entre la preeclamsia y la } \\
\text { PC encontrada en el presente estudio, hay escasa litera } \\
\text { tura. Un estudio de cohortes realizado en base al registrc } \\
\text { Noruego de PC encontró un exceso de riesgo de PC er } \\
\text { hijos de madres con preeclampsia. En otro estudie, la } \\
\text { asociación de preeclamsia y PC estaba mediada por la }\end{array}$} \\
\hline
\end{tabular}


Tabla 4. Análisis univariado y multivariado de los factores de riesgo perinatales

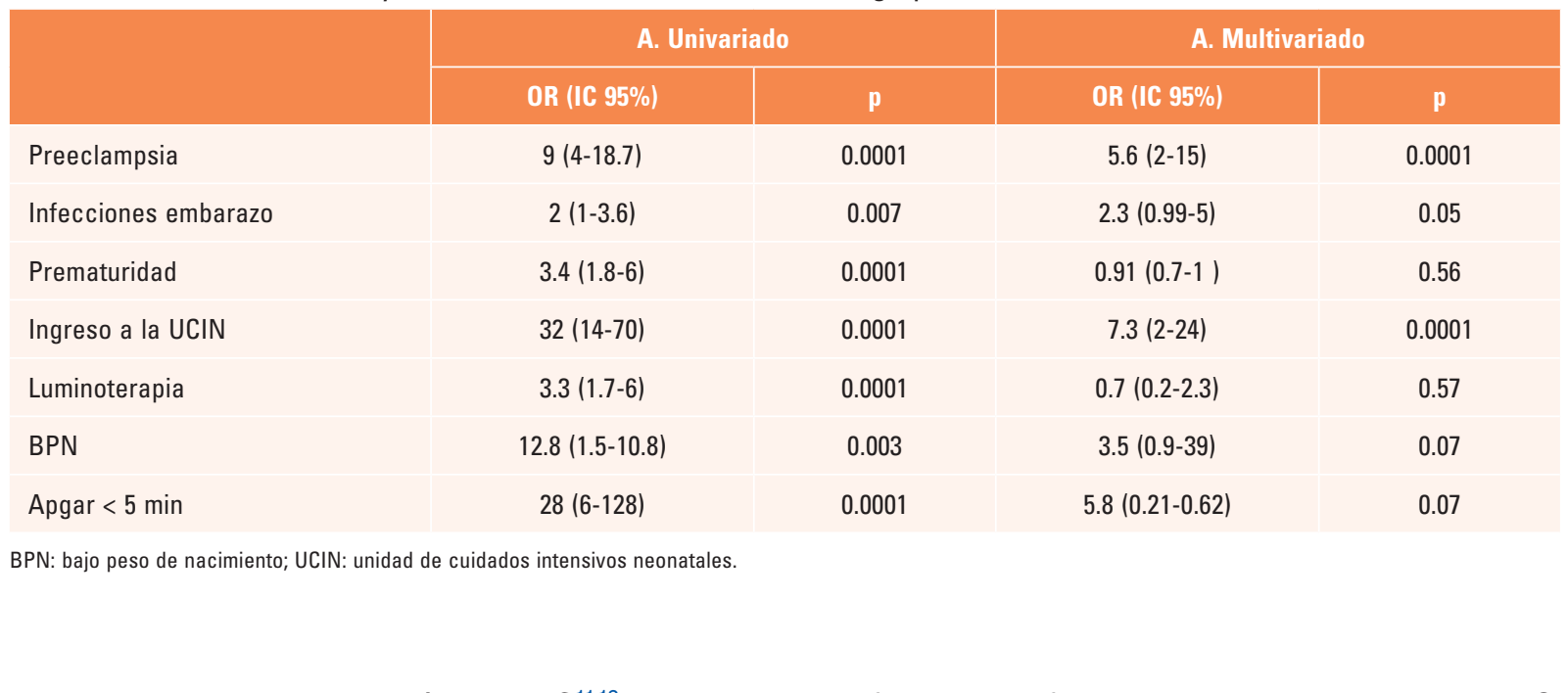

prematuridad y los nacidos de término PEG ${ }^{11,12}$. En el presente reporte, los antecedentes de BPN y prematuridad se asociaron a PC en el análisis univariado, pero no en el muitivariado. Es posible que el escaso número en cada grupo haya influido. Por el mismo motivo, los participantes con antecedentes de MBPN y PEG no fueron incluidos en el análisis de regresión. Por otro lado, tanto la población de prematuros como la de BPN no eran muy pequeños, sino moderados (prematuros grandes y peso de nacimiento que se aproximaba más a 2,500 g que a $1.500 \mathrm{~g}$ ).

La preeclamsia es responsable del $18 \%$ de todas las muertes maternas en todo el mundo y se asocia a secuelas neurológicas no solo en la mujer sino también en sus hijos. La incidencia global varía de un país a otro y está entre el 2 y el 3\%13,14. La inflamación materna en la preeclamsia desempeña un papel importante en el desarrollo neurológico del feto. Durante la placentación se desarrollan procesos inflamatorios considerados normales, sin embargo, en la preeclamsia se produce un estado de inflamación con mayor liberación de citocinas, como las interleucina (IL-6) y el factor de necrosis tumoral (TNF- $\alpha)$, relacionadas a las alteraciones del sistema nervioso central en desarrollo ${ }^{15}$. La enfermedad hipertensiva del embarazo, y específicamente el subgrupo de preeclampsia, se asoció a riesgo aumentado de trastorno del espectro autista en los productos ${ }^{16}$. Otras alteraciones neurológicas, sensoriales y cognitivas también se han relacionado con la preeclamsia ${ }^{17}$. Un estudio de cohortes llevado a cabo en Canadá encontró mayor porcentaje de alteraciones del neurodesarrollo en hijos de madres con preeclamsia ${ }^{18}$.

El otro factor asociado a PC encontrado en este estudio fue la hospitalización en la UCIN. Si bien el mayor ingreso a la UCIN correspondió a hijos de madres con preeclamsia, fue un factor de riesgo independiente y un modificador del efecto de la preeclamsia sobre la La causa prevalente de ingreso a la UCIN fue la dificultad respiratoria. El ingreso a la UCIN expone al neoñato a las infecciones nosocomiales, generalmente ${ }_{\mathrm{j}}^{\mathrm{j}} \mathrm{pr}$ gérmenes multirresistentes. La sepsis en el periodo neonatal se asocia a alteraciones del neurodesarióllo, entre ellos la $\mathrm{PC}^{19}$. Por otro lado, las infecciones perinatales, como la corioamnionitis y la infección ypor citomegalovirus (CMV), exponen a los neonatos $\mathrm{a}$ infecciones graves, ingreso a la UCIN y a mayor riesgo de PC. El conocimiento del papel de los mediadores inflamatorios en la fisiopatología de las alteraciones del neurodesarrollo permitió identificar a IL 17A como una sustancia proinflamatoria en la corioamnionitis materna, con potencialidad de producir daño neurológico ${ }_{-}^{2 \theta-22}$. Las infecciones maternas recogidas en este estudio correspondieron en más de la mitad a infeccionesurinarias durante el embarazo, un caso de CMV, y dos casos de toxoplasmosis. Aunque no se encontró âsociación con PC en el presente trabajo, su papel amêrita de más estudios.

En países en vías de desarrollo, los recursos ensalud son escasos, hay poca inversión en cuidados intensivos neonatales y escasa información sobre el seguimiento de estos pacientes. En el 2015, Milner, et al. publicaron los resultados de una revisión sistemática de las secuelas de pacientes neonatos y lactañtes pequeños sobrevivientes de las salas de cuidãdos intensivos en países en vías de desarrollo. La prematuridad y el BPN se asociaron a un $11.2 \%$ con PG, la asfixia perinatal a un $22.8 \%^{23}$.

Una de las secuelas de la asfixia perinatal acompañada de encefalopatía hipóxico- isquémica graves es la $\mathrm{PC}^{24}$. Aunque un grupo de pacientes tenían el diagnóstico de asfixia perinatal, no se incluyeron en el análisis 
porque al ser un dato retrospectivo no se pudieron corroborar los criterios diagnósticos considerados.

El estudio tiene las limitaciones de la recolección de datos perinatales en forma retrospectiva. Tampoco se recogieron datos que permitan clasificar el grado de preeclampsia con certeza. Los resultados no pueden generalizarse a toda la población por ser un estudio realizado en un solo hospital. Sin embargo, aporta información sobre la asociación entre la preeclamsia, el egreso de la UCIN y la PC, causante de discapacidad irreversible. Es posible que los principales factores de riesgo de $\mathrm{PC}$ difieran en función de la prevalencia de los factores de riesgo perinatales en las regiones 0 poblaciones. En el país donde se realizó el estudio, la preeclamsia es prevalente y la tercera causa de mortalidad materna. En algunos países de África, la PC está ligada a la infección perinatal por el $\mathrm{VIH}^{25}$. Este estudio puede constituirse en punto de partida de uno más extenso y prospectivo. El mayor conocimiento de la relación entre estas patologías perinatales puede llevar a la implementación de intervenciones sanitarias preventivas tanto en el control prenatal, parto y manejo de la preeclamsia, como en estrategias de neuroprotección de los neonatos hospitalizados en la UCIN. Es importante el seguimiento de los hijos de madres con preeclamsia con el objetivo de detectar en forma temprana las alteraciones del neurodesarrollo.

\section{Conflicto de intereses}

Los autores declaran no tener conflicto de intereses.

\section{Responsabilidades éticas}

Protección de personas y animales. Los autores declaran que para esta investigación no se han realizado experimentos en seres humanos ni en animales.

Confidencialidad de los datos. Los autores declaran que han seguido los protocolos de su centro de trabajo sobre la publicación de datos de pacientes.

Derecho a la privacidad y consentimiento informado. Los autores han obtenido el consentimiento informado de los pacientes y/o sujetos referidos en el artículo. Este documento obra en poder del autor de correspondencia.

\section{Bibliografía}

1. Baxter $P$, Morris $C$, Rosenbaum $P$, Paneth $N$, Leviton A, Goldstein M, et al. The Definition and Classification of Cerebral Palsy. Dev Med Child Neurol. 2007; Suppl:1-44.
2. Oskoui M, Coutinho F, Dykeman J, Jetté N, Pringsheim T. An update on the prevalence of cerebral palsy: A systematic review and meta-analysis. Dev Med Child Neurol. 2013;55(6):509-19.

3. Mclntyre S. The continually changing epidemiology of cerebral palsy. Acta Paediatr Int J Paediatr. 2018;107(3):374-5.

4. Mclntyre S, Taitz D, Keogh J, Goldsmith S, Badawi N, Blair E. A systematic review of risk factors for cerebral palsy in children born at term in developed countries. Dev Med Child Neurol. 2013;55(6):499-508.

5. Fahey MC, Maclennan AH, Kretzschmar D, Gecz J, Kruer MC. The genetic basis of cerebral palsy. Dev Med Child Neurol. 2017;59(5):462-9.

6. Lien E, Andersen G, Bao Y, Gordish-Dressman H, Skranes JS, Blackman JA, et al. Genes determining the severity of cerebral palsy: The role of single nucleotide polymorphisms on the amount and structure of apolipoprotein e. Acta Paediatr Int J Paediatr. 2015;104(7):701-6.

7. UNICEF. Situación del Derecho a la Salud. Materna,Infantil y Adolesscente en Paraguay. 2013. p. 116.

8. Arora NK, Nair MKC, Gulati S, Deshmukh V, Mohapatra A, Mishka D, et al. Neurodevelopmental disorders in children aged 2-9 years: Population-based burden estimates across five regions in India. PLoSYMed. 2018;15(7):1-19.

9. Yuan J, Wang J, Jieqiong MA, Zhu D, Zhang Z, Li J. Paediatric cerebral palsy prevalence and high-risk factors in Henan Province, central China. J Rehabil Med. 2019:51(1):47-53.

10. Sellier E, Platt MJ, Andersen GL, Krägeloh-Mann I, De La Cruz J, Căns C, et al. Decreasing prevalence in cerebral palsy: A multi-site European population-based study, 1980 to 2003. Dev Med Child Neurol. 2016;58(1):85-92.

11. Strand KM, Heimstad R, Iversen AC, Austgulen R, Lydersē $S$, Andersen GL, et al. Mediators of the association between pre-eclampsia and cerebral palsy: population based cohort study. BMJ [Internet]. 2013;347:1-10. Disponible en: http://dx.doi.org/doi:10.1136/bmj.f4089

12. Blair $E$, Watson $L$. Cerebral palsy and perinatal mortality after pregnancy-induced hypertension across the gestational age spectrum: Observations of a reconstructed total population cohort. Dev Med Child Neurol. 2016;58:76-81.

13. ljomone OK, Shallie $P$, Naicker T. Changes in the structure and function of the brain years after Pre-eclampsia. Ageing Res Rev [Internet]. 2018;47(July):49-54. Disponible en: https://doi.org/10.1016/j.arr.2018.06.006

14. Abalos E, Cuesta C, Carroli G, Qureshi Z, Widmer M, Vogel JP, cet al. Pre-eclampsia, eclampsia and adverse maternal and perinatal outcomes: a secondary analysis of the World Health Organization Multicountrysurvey on Maternal and Newborn Health. BJOG. 2014;121 Suppl:14-24.

15. Maher GM, McCarthy FP, McCarthy CM, Kenny LC, Kearney PM, Khashan AS, et al. A perspective on pre-eclampsia and neurodevelopmental outcomes in the offspring: Does maternal inflammation play a role?-Int $\mathrm{J}$ Dev Neurosci [Internet]. 2018; Disponible en: https://doi.org/10.1016/.ijdevneu.2018.10.004

16. Maher GM, O'Keeffe GW, Kenny LC, Kearney PM, DinanTG, Khashan AS. Hypertensive disorders of pregnancy and risk of neधrodevelopmental disorders in the offspring: A systematic review and meta-analysis protocol. BMJ Open. 2017;7(10):1-5.

17. Rätsep MT, Hickman AF, Maser B, Pudwell J, Smith GN, Brien D, et al. Impact of preeclampsia on cognitive function in the offspring. Behav Brain Res [Internet]. 2016;302:175-81. Disponible en: http://dx.doi.org/10.1016/j.bbr.2016.01.030

18. Warshafsky C, Pudwell J, Walker M, Wen SW, Smith GN. Prospective assessment of neurodevelopment in children following a pregnancy complicated by severe pre-eclampsia. BMJ Open. 2016;6(7):1-7.

19. Le Doare K, Dangor Z, Sobanjo-ter Meulen A, Russell NJ, Bartlett L, Rattue $\mathrm{H}$, et al. Neurodevelopmental Impairment in Children After Group B Streptococcal Disease Worldwide: Systematic Review and Meta-analyses. Clin Infect Dis. 2017;65(suppl_2):S190-9.

20. Dakovic I, Da Graça Andrada M, Folha T, Neubauer D, Hollody K, Honold M, et al. Clinical features of cerebral palsy in children with symptomatic congenital cytomegalovirus infection. Eur J Paediatr Neurol. 2014;18(5):618-23.

21. Bear JJ, Wu YW. Maternal infections during pregnancy and cerebral palsy in the child. Pediatr Neurol. 2016;57:74-9.

22. Lawrence SM, Wynn JL. Chorioamnionitis, IL-17A, and fetal origins of neurologic disease. Am J Reprod Immunol. 2018;79(5):e12803.

23. Neal EFG, Steer AC, Duke T, Roberts G, Milner KM. Long-term neurodevelopmental outcome in high-risk newborns in resource-limited settings: a systematic review of the literature. Paediatr Int Child Health. 2015;35(3):227-42.

24. Polin RA, Randis TM, Sahni R. Systemic hypothermia to decrease morbidity of hypoxic-ischemic brain injury. J Perinatol. 2007;27(Suppl. 1):47-59.

25. Johnson A, Khurana E, Westmoreland KD, Gambrah-Sampaney C Bearden DR, Mazhani L, et al. Risk Factors for Cerebral Palsy in Children in Botswana. Pediatr Neurol [Internet]. 2017;77:73-7. Disponible en: https:// doi.org/10.1016/j.pediatrneurol.2017.07.014 